\title{
Data Model and Application of Pipeline Integrity Management
}

\author{
Bingtao Dai \\ Langfang CNPC Lang Wei Engineering Project Management Co., Ltd., Langfang, Hebei, 065000 China
}

\begin{abstract}
Data is an important foundation and premise to ensure pipeline integrity management. Using data model to manage and utilize data is the key to implement pipeline integrity management, which can not only ensure the safety of oil and gas transportation, but also promote the stable development of China's social economy. Based on this, this paper deeply analyzes the development status of oil and gas pipeline integrity management data model, and makes an in-depth exploration on the establishment and application of oil and gas pipeline integrity data model by comparing various data models, combined with the current situation of oil and gas pipeline integrity management, and with the help of the advantages of apdm model, such as data set division and spatial information management.
\end{abstract}

Keywords: Oil and gas pipeline, Integrity management data model, Establishment process.

\section{Introduction}

For a long time, oil and gas pipelines have undertaken important energy transportation tasks. However, because oil and gas products belong to high-risk transportation media, and the geological conditions of pipeline route areas are complex, and it is difficult to maintain in some areas of the route, the safety of oil and gas pipelines has become one of the topics focused on by countries all over the world. At the same time, oil and gas pipeline engineering involves many disciplines, and has the characteristics of many pipeline basic data, complex information types and sources, and dynamic real-time update of operation information. Therefore, the rationality and accuracy of data processing mechanism can directly affect the subsequent identification and evaluation results. In this situation, it is necessary to build a more scientific and reasonable data model with the help of the pipeline integrity management concept and implementation criteria proposed by API and ASME. So as to further strengthen the data management and utilization of oil and gas pipelines and ensure the safe operation of oil and gas pipelines.

\section{Development status of data model of pipeline integrity management}

The certainty of data model can provide guarantee for data sharing and information integration. For oil and gas pipelines, the composition of pipeline system is complex (see Figure 1), and there are many kinds of data information involved. Including specific pipeline facilities, operation and maintenance, internal and external inspection, hydrogeology and spatial data, etc.
Therefore, it is necessary to establish a data model with reasonable construction, strong pertinence and wide application range to manage and display the data. At present, the existing pipeline models mainly include ISAT, Smallworld, ISPDM, PODS, APDM, etc. Among them, Integrated Spatial Analysis Technology (ISAT) was an earlier oil and gas pipeline data model in 1977, which specifically includes pipeline facilities, crossing and related pipeline events, and has been basically replaced by PODS. The Smallworld data model integrates applications based on the Long-distance Pipeline Management Solution (GTO). The GTO has also become part of the Smallworld software solution. Industrial Standardized Pipeline Data Management (ISPDM) is designed according to the relevant standards of the European pipeline industry sector, not only integrating data warehouse and Web technology. At the same time, multiple modules such as inspection, maintenance process and risk are also introduced, which can provide users with the function of three-dimensional modeling [1].

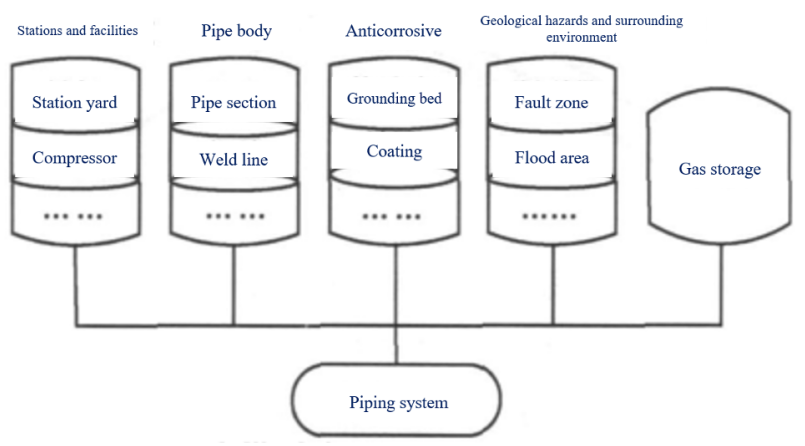

Figure 1 Composition of piping system 
Pipeline Open Data Standard (PODS), which covers all pipeline management services, is a relational data model specially designed for the application management of oil and gas pipeline technology system. ISAT is the main design source of this model, which can provide a completely open database modeling platform for society. At the same time, in the process of the gradual development of pods model, the design of database standardization, pipeline business expansion and geographic information application has also been further improved, and the connectivity and robustness of the system have been significantly enhanced, which has been recognized and applied by the majority of pipeline operators. For example, in pods6.0, the model has covered 30 classification modules such as environmental information, pipeline repair, internal and external detection and geographical entities, including more than 700 data tables and more than 5800 data items.

ArcGIS pipeline data model (apdm) is a pipeline data model developed on the basis of Geodatabase technology of ESRI company. Among them, apdm5.0 model specifically covers 9 data classification sets, such as cathodic protection, centerline and hierarchy, instrument reading, equipment, operation, integrity and event support [2], which can greatly enhance the flexibility of data template operation. At the same time, operating enterprises can flexibly formulate and implement models according to specific business requirements, and can also improve the models by modifying and adding existing elements, so as to realize the interoperability between database management and pipeline business as soon as possible. In addition, the advantages of APDM are mainly reflected in the process of connecting pipe spatial data with attribute data, and it also supports the 3D analysis function related to GIS in specific applications. At present, there have been a large number of successful application cases of APDM at home and abroad, and this model has been recognized by the pipeline industry. This paper will focus on analyzing and researching the integrity data management model based on APDM.

\section{Establishment of data model of pipeline integrity management}

At present, the data set of oil and gas pipeline integrity data model is very complex, mainly including centerline and hierarchy, facilities along the line, basic geography, operation, pressure, cathodic protection, crossing and third-party damage, integrity detection and evaluation, identification and risk evaluation of high consequence areas, efficiency evaluation, etc. In total, there are more than 190 items in the pipeline data sheet, including more than 60 items referencing and modifying the original apdm structure sheet and more than 130 items creating new sheets. Therefore, this model not only completely retains the core classes of apdm, but also expands and modifies the optional classes. According to the specific requirements of integrity management, it focuses on the construction of relevant data structures such as occupation, high consequence area identification and risk evaluation, cross and third-party destruction, integrity detection, evaluation and efficiency evaluation [3].

Affected by many factors, such as poor natural environment, unreasonable urban planning, third-party damage, complex interleaving and parallel of municipal facilities and pipelines, oil and gas pipeline failure accidents occur frequently, and most of them occur in economically developed and densely populated areas. The data set of "Occupy, Intersection and Third Party Destruction" in the oil and gas integrity data model is based on the infringement data in APDM, which adds records of many common pipeline hazards such as occupy, illegal drilling and excavation destruction, and further expands the classification of municipal facilities, such as fire-fighting pipelines, sewage pipelines, cable optical fibers and railways, etc., constructs a global attribute domain, and clearly marks the elements of municipal facilities lines.

ASME and API have made clear provisions on the relevant requirements for the original data of potential influencing factors in high consequence areas. Among them, pipeline risk management (see Figure 2 for the specific process) and evaluation, as a key link of oil and gas pipeline integrity management, should comprehensively analyze and consider the identification of risk sources, selection of evaluation models, rules for pipe section division, failure consequences, risk factor tree level and other factors. At the same time, it is also necessary to carry out detailed analysis and improvement on the high-consequence areas, sections of highconsequence areas, the scope of high-consequence areas and risks in operation categories in APDM supervision category, refine the classification indicators of identification standards, and divide the main risk factors into design and construction, corrosion, excavation damage, operation and maintenance, deliberate damage and natural disasters, etc., so as to provide comprehensive and reliable basis for risk segmentation, such as soil type, mileage, regional grade, anticorrosive coating and station.

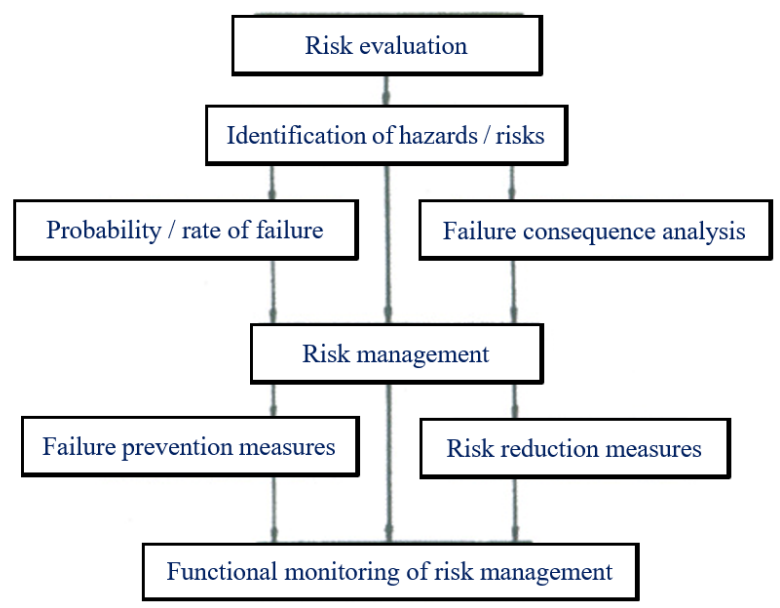

Figure 2 Risk management process

At this stage, the most intuitive way to distinguish and analyze pipeline defects is pipeline inspection. At the same time, the accuracy of pipeline inspection data directly affects the credibility and quantification of the 
integrity evaluation, providing a valuable reference for the maintenance of oil and gas pipelines. For APDM, the detection categories specifically include anomalies, closely spaced potential measurement readings, anomaly clusters and detection ranges, but there is no clear division of defect types [4]. Therefore, it is necessary to build the "Integrity Testing and Evaluation" data set, refine the internal testing data, classify and mark the pipe body dents, manufacturing defects, metal losses and weld defects (longitudinal, circumferential and spiral), increase various evaluation information such as corrosion stress analysis, defect analysis and durability analysis, and also include the input of the results of external testing methods such as DCVG technology of corrosion protection layer damage detection and ground magnetic leakage.

In addition, as an important hub for the development, storage and transportation of offshore oil and gas fields, the operating environment of submarine pipelines is more complicated, and the detection and maintenance is more difficult, and the requirements for environmental data in integrity management are more stringent, requiring detailed data records for tidal level period, geological sampling-static exploration coordinates and other aspects [5]. In the application, the design objects of apdm are only aboveground pipeline system and buried pipeline system, so there is no in-depth thematic expansion for submarine pipeline and offshore facilities. Therefore, it is necessary to establish a submarine pipeline data set to objectively and accurately predict the specific trend of soil foundation evolution by collecting relevant environmental information, such as tide level, water depth, ocean current, soil mechanics index, sediment concentration and seabed topographic coordinates. The requirements for facilities along the submarine pipeline and cathodic protection The point elements on the center line are mainly located by mileage, the offline point elements are mainly located by the conversion of GPS coordinates (x.y, z) or the distance from the nearest reference target, while the line elements are mainly It locates through the interval of known mileage [7]. The facilities along the pipeline include valves, girth welds, fixed piers, anti-corrosion coatings and hydraulic protection, etc., as well as various activities.

\section{Implementation and application of data model for oil and gas pipeline integrity management}

Geodatabase spatial database lays a good modeling foundation for lidm model, and still uses the behavior and relationship in apdm abstract classes. As the object class, relation class and feature class in APDM, the core class can clearly point out the expression rules of elements along the oil and gas pipeline model and centerline features with the help of unified coordinate system types and reference attributes [6].

Station location, three pipelines and PS datum point are the core elements of the model. In specific applications, GIS focuses on the absolute positioning of pipeline positioning points, and other relative positioning points can be easily calculated after the station values are clear and quantified. Columns are represented by line feature classes, and there are three sub-classifications (subtypes), including level, mileage pile and continuity. The central point elements are located mainly by mileage, while the offline point elements are located mainly by the conversion of GPS coordinates (x.y, z) or by the distance from the nearest reference target, while the line elements are located mainly by the known mileage interval [7]. Facilities along the pipeline specifically include various elements such as valves, girth welds, fixed piers, anticorrosive coatings and hydraulic protection, as well as various activities. For example, elements such as cathodic protection, line change, pipe change, monitoring and emergency can be superimposed on the center line of oil and gas pipeline, and a dynamic linear reference corresponding to the abstract relationship can be established. The level of pipeline events is mainly divided by the pipe network and subsystems. Among them, the pipe network is divided by creating geometric networks, and the subsystems are divided by thematic classification and plane topology. However, in the oil and gas pipeline integrity management data model, the identification attribute of any event is unique. Therefore, it should be clear that the feature class or object class does not depend on the classification. Therefore, it should be distinguished by main and foreign keys to enhance the relationship between feature class and object class.

The application of oil and gas pipeline integrity management data model to oil and gas pipeline integrity management has the following advantages: first, Geodatabase spatial database technology can realize the integrated management of pipeline construction, spatial data and operation data, and greatly improve the accuracy of data entry and editing through attribute verification and global attribute domain setting, Reduce operational errors. Secondly, through the expression of topological space, we can define the characteristics of environmental elements, event elements and pipeline elements, and at the same time, we can also define the relationship among the elements, so that the elements can make corresponding response according to the specific changes of the specified elements, and effectively realize the dynamic cascade of pipeline information [8]. Third, in the process of oil and gas pipeline integrity management, the relationship between data collection classification and level undertaking should be improved, so as to enhance the purpose of data collection, improve the rationality of evaluation process, and lay a good foundation for thematic data extraction, data warehousing and the construction of integrity data warehouse. Fourth, because the data model of oil and gas pipeline integrity management has strong expansibility, it can meet the long-term application needs of oil and gas pipeline integrity management.

\section{Conclusions}

To sum up, the integrity management of oil and gas pipelines is very complicated, with strong overall situation and high data quality requirements. Therefore, only by building a unified data model and formulating reasonable data collection standards can we ensure the 
smooth development of the integrity management of oil and gas pipelines. At the same time, in order to further improve the effectiveness and standardization of oil and gas pipeline data management, in addition to establishing lidm data model, it is also necessary to improve the existing pipeline data classification methods and collection forms, actively improve the construction methods of data set data structures such as infringement, identification, evaluation and submarine pipelines, and deeply explore the methods and methods of oil and gas pipeline integrity data management, So as to ensure the safe operation of oil and gas pipelines.

\section{References}

1. He Wang, Xie Liyun, Wei Ziyu. Evaluation of engineering geological suitability of long-distance natural gas pipeline project crossing areaComment on "Oil and Gas Pipeline Integrity Evaluation Technology" [J]. Xinjiang Geology, 2019, 37(4): Post 4.

2. Wang Qingguo, Guo Min. Research on the influencing factors of gas pipeline integrity based on ISM and AHP[J]. Gas and Heat, 2019, 39(4): Postinsertion 1- Post-insertion 4.

3. Ma Bin, Jiang Feng, Ma Xuqing. Integrity evaluation technology of defective pipelines[J]. Gas and Heat, 2019, 39(5): 32-39.

4. Cui Ruixue, Wang Lin, Zhao Deyin, et al. Research on technology development of pipeline integrity management platform[J]. Contemporary Research in Chemical Industry, 2020(11): 108-110.

5. Jin Jian, Zhu Xueshan, Liu Yanyang, et al. Research on big data integration technology methods for oil and gas long-distance pipelines[J]. Oil and Gas Field Surface Engineering, 2020, 39(2): 77-81.

6. Jiang Zhongan, Zheng Dengfeng, Zeng Fabian, et al. Research on the safety management model of oil and gas pipelines based on hazard theory [J]. Journal of Hunan University (Natural Science Edition), 2021, 48(4): 56-65.

7. Jia Shuai. The application of intelligent information technology in the integrity construction of the Beijing-Tianjin Second Oil Pipeline[J]. Oil and Gas Field Surface Engineering, 2021, 40(2): 97-102.

8. Hou Lina, Li Yuanpeng, Yan Wei, et al. Construction and fusion method of oil and gas field pipeline and station integrity management system $[\mathrm{J}]$. Oil and Gas Field Surface Engineering, 2021, 40(3): 63-69. 